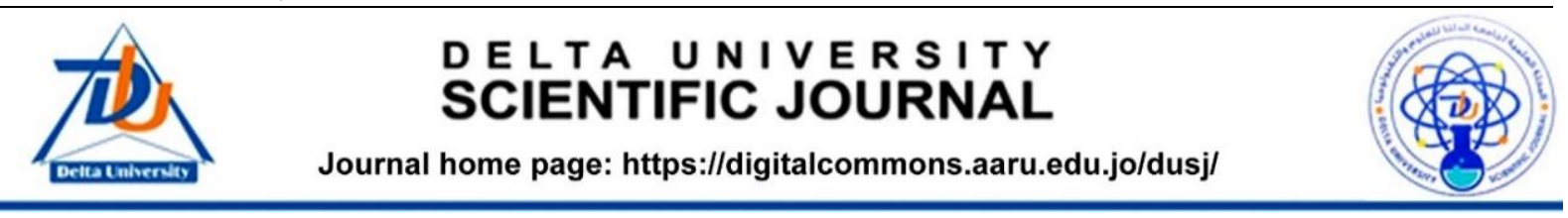

\title{
MIS and Risk Management during the knowledge technics on Algerian universities :Bordj Bou Arreridj University case study
}

\section{Benharira Nadjet *1}

1 Teaching assistant B, Department of Human and Social Sciences, Mohamed Khieder Biskra University, Algeria.

\section{Keywords:}

1- Key Artificial intelligence

2- BBA University

3- Management Information

Systems

4- Risks

Management

5- Smart

Information

Systems

\begin{abstract}
The primary aim of the subject was to study the impact of MISs accredited in Algerian universities on managing the risks they face, especially during the knowledge technologies that the world is witnessing, by relying on the descriptive and analysis methodology to conduct the field study through the Questionnaire tool directed to administrators and employees working in the Faculty of Human and Social Sciences at the University of BBA to answer the following problem: What is the role that MISs play in risk management in Algerian universities? What is the future and prospects for risk management during the knowledge technologies? The subject was studied based on three basic frameworks, the first one represented the methodological aspect of the study to determine the most important variables of the topic and its scientific direction ; and the second framework which dealt with a set of basic concepts about the study, and identify about the role of MIS in facing risks and its effectiveness, in addition, the role of information technology in the risk tool, and the most important information requirements for decision-making during risks, besides that, the role of $\mathrm{AI}$ in supporting and improving decision-making in the field of higher education and scientific research, as well as, modern approaches to knowledge-based expert information systems technologies, and finally the piratic framework which included several basic elements about the role of MIS in risk management during the Corona pandemic, in addition to studying the prospects and future of risk management through knowledge technics, then arriving at results and suggestions.
\end{abstract}

$$
\text { نظم المعلومات الإدارية و إدارة المخاطر في ظل تقنيات المعرفة بالجامعات الجز ائرية: در اسة ميدانية }
$$

1 بن حريزة نجاة

1 ملرس مريرة نجاعد ب، قسم العلوم الإنسانية والاجتماعية، جامعة محمد خيضر بسكرة، الجزائر

$$
\begin{aligned}
& \text {-1نظم المعلومات } \\
& \text { الإدارية } \\
& \text { 2-2 إدارة المخاطر } \\
& \text { 3-تقنيات المعرفة } \\
& \text {-4الذكاء الاصطناعي - } \\
& \text { 5-5نظم المعلومات الذكية }
\end{aligned}
$$

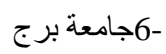

$$
\begin{aligned}
& \text { بو عريريج }
\end{aligned}
$$

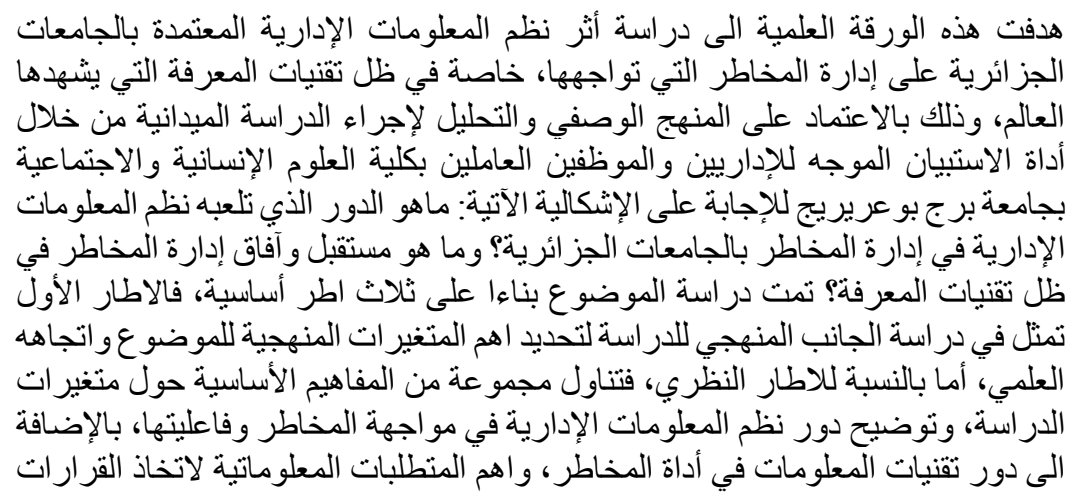




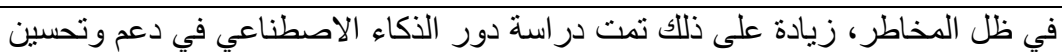

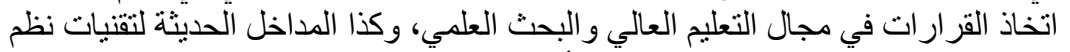

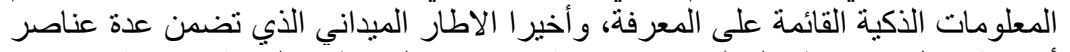

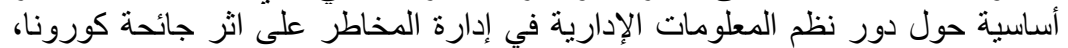
بالإضافة الى دراسة آفاق ومستقبل إدارة المخاطر من خلال تقنيات المعرفة المارة، ثم التوصل

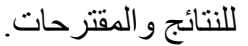

في إدارة المخـاطر و الكوارث التي تواجهرهـا الموارد

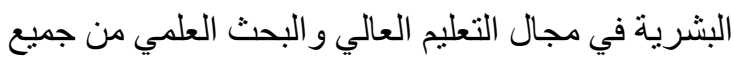
نو احيـه التعليميـة، العلميـة و الإداريـة وحتى البيداغوجيـة منها، فإدارة المخاطر تتطلب وضع البدائل المقترحة لحل لحل المشـــكلات و التغلب على اهم الصـــعوبات التي نواجها

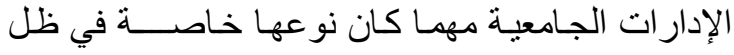
التقنيات الحديثة التي تسـاهم في خلق فرص اكثر لإدارة المخاطر بأقصــر وقت وجهذ ممكنين، وتمكن من زيادة

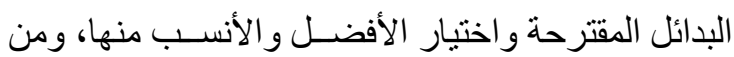
جهة أخرى فنجد أن هذه التقنيات تطرح عدة تعقيدات

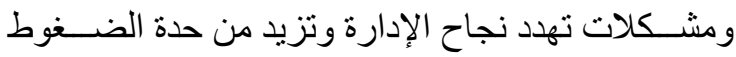
المهنية، ومن هنا نطرح التساؤل التالي: ماهو الدور الذي تقوم بـه نظم المعلومـات الإداريـة في إدارة المخـاطر بـالجامعـات الجزائريـة؟ ومـا هو مســتقبل وآفاق إدارة المخاطر في ظل تقنيات المعرفة؟

$$
\text { 3. }
$$

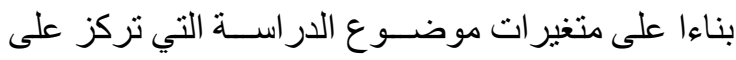
دور نظم المعلومـات الإداريـة في إدارة مخـاطر إدارات الجامعات الجزائرية في ظل تقنيات المعرفة، فان هذه الدر اسـة هدفت الى تحقيق جملة من الأهداف المتمثلة في مايلي:

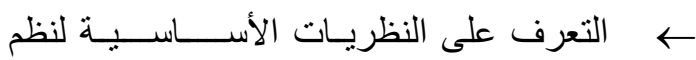
المعلومات الإدارية و إدارة المخاطر. — قياس أثر اســتخدام نظم المعلومـات الإداريـة بالإدار ات الجامعية الجز ائرية.

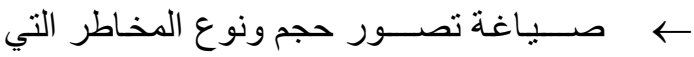
تو اجهها الإدارات الجامعية الجز ائرية بصفة روتينية

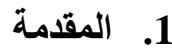

تعد تحتل نظم المعلومات الإدارية موقعا هاما ضمن الهيكل التنظيمي للإدار ات بالجات الإدامعات الجز ائرية،

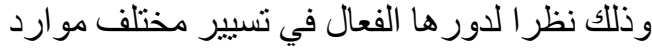

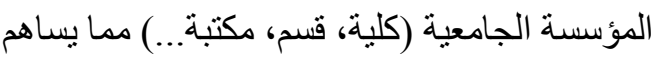

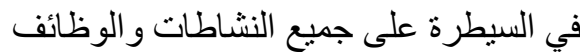

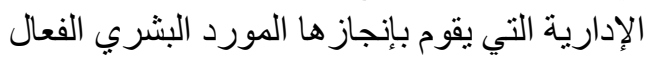

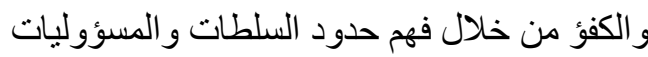
و اتخاذ القرار ات المناسبة والوقوف على نقاط نقاط القوة و والضعف بهذه الجامعات. لكن وفي بعض الأحيان نلاحظ أن الإدارة الجامعية تتعرض لمجموعة من المخاطر التي تهدد نجاحها، ممـا يؤدي الى زيـادة الأعبـاء الإداريـة ونقص فرص تحليل

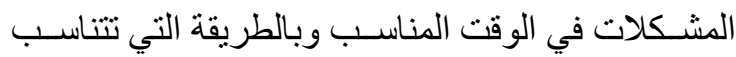

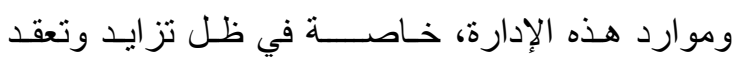
الاحتيـاجـات العلميـة و التكوينيـة و المعر فيـة بـالجـامعـات الجزائرية في الوقت الذي ظهرت فيه ما يســمى بتقنيات المعرفة وتضـــم حجم البيانات وتعقد طرق تســبير ها و الخروج بالقر ار ات المناسبة، خصوصـا خلال السنوات

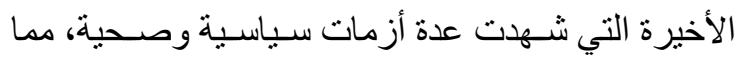

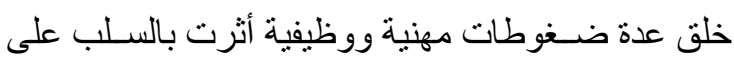

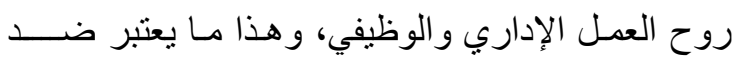
مصلحة الجامعة الجز ائرية.

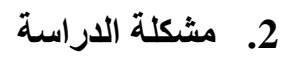

تقوم نظم المعلومـات الإداريــة بعـدة أدوار وظيفيـة بالجامعات الجزائرية و المتمثلة في التخطيط الاستر اتيجي لنظم التعليم العالي و البحث العلمي، بالإضـــافة الى تبني

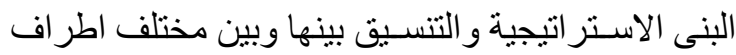
العلميـة التعليمة في معظم الكليـات و المعـاهد، نهيك عن لهن التنظيم و التوجيـه لمختلف البـاحثين و الجـامعيين للموارد التعليمية الحديثة و التكنولوجية، هذا ويظهر دور ها أيضـا 


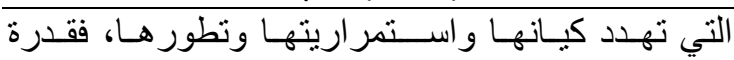

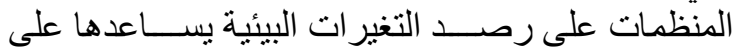

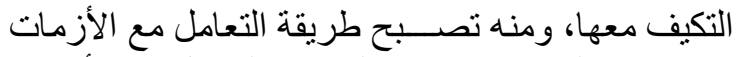
امر هين، ولكي تســنـيع المنظمة التعامل مع الأزمات التهات

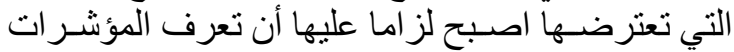

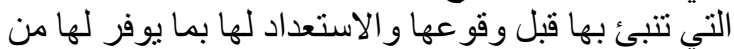

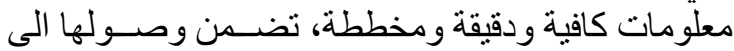
متخذ القرار في الوقت المناسب.

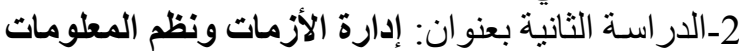

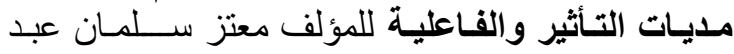

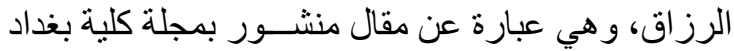

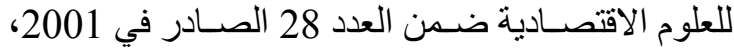

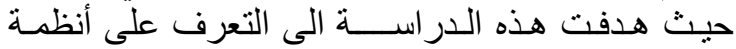

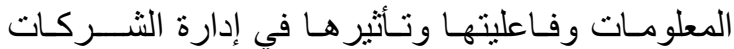

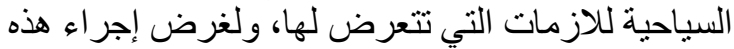

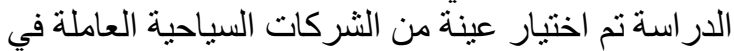

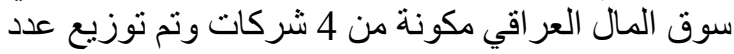

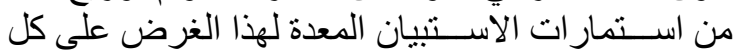

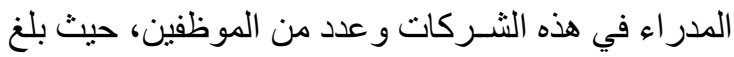

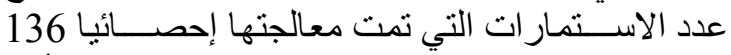

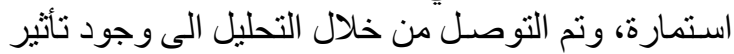

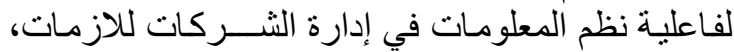

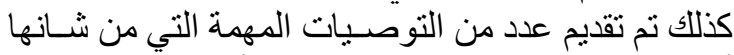

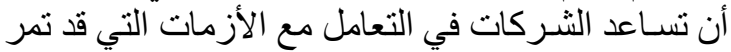

بها في مسيرة عملها.

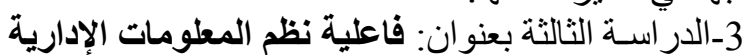

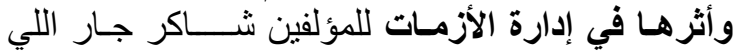

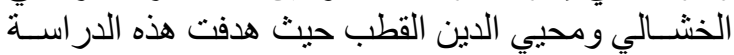

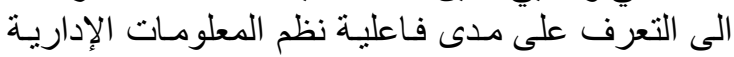

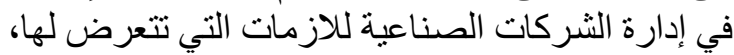

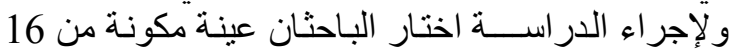

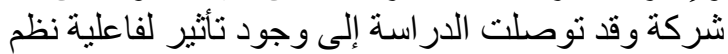

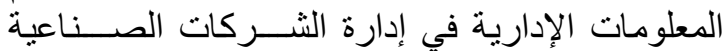

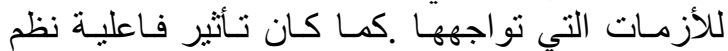

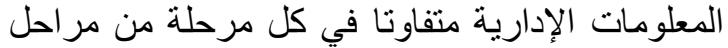

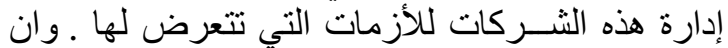

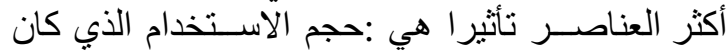

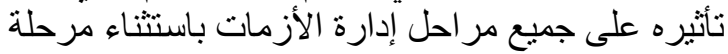

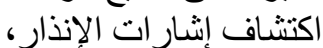

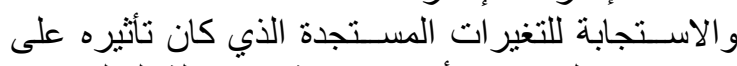
جميع مر احل إدارة الأزمات باستثناء مرحلة التعلم.

\section{5.}

بناءا على موضوع الدور استة، فانه تم الاعتماد على المنهج

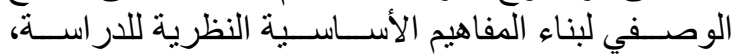

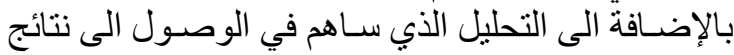

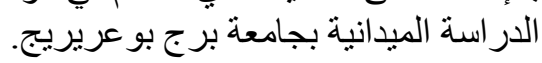
6 من خلال ما تقدم، نتوصــــل الى مجمو عة من النتائج التي

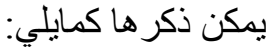

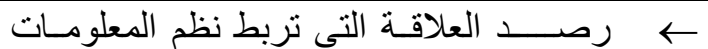

الإدارية بإدارة المخاطر في مجال التعليم العالي و البحث<smiles>[AlH2]=[AsH2]</smiles>

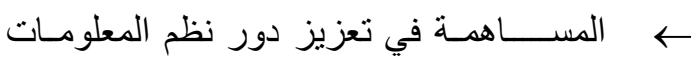

الإداريـة في التخفيض من حـدة المخــاطر من خلال

استعمالات تقنيات المعرفة

— استكثــاف المبررات المتعلقة باستخدام تقنيات

المعرفة في مجال الذكاء الاصــــناعي ونظم المعلومات

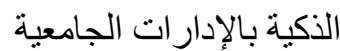

— مقارنة البدائل و الحلول الكفيلة بإدارة المخاطر

باستخدام تقنيات نظم المعلومات الذكية.

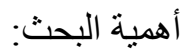

تتخذ الدر اســة أهميتها من أهمية الموضــوع الذي تنتاولته و المتعلق بدر اســــة الأدوار الحديثـة التي تقوم بهـا نظم

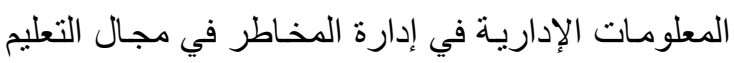

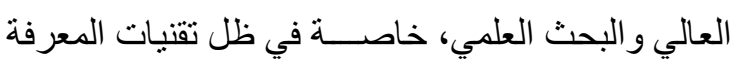
الحديثة، وتكمن أهمية الدراسة في إبراز العلاقة الوظيفية بين نظم المعلومات الإدارية و النظم الخبيرة وكذا تقنيات الذكاء الاصـطناعي، فهذه الدر اسـة تعتبر بمثابة إضـافة لمجال العلوم الإنســـانية و الاجتمـاعية و إدارة المكتبات ومر اكز التوثيق، هذا بالإضـافة الى أنها تسـهم في معالجة بعض القضـايا العالقة بالإدار ات الجامعية خاصـة في ظل

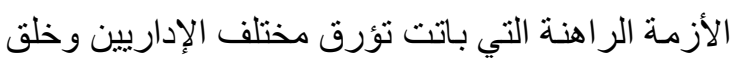

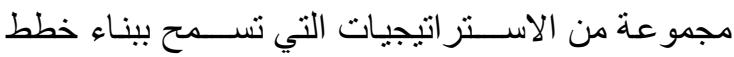

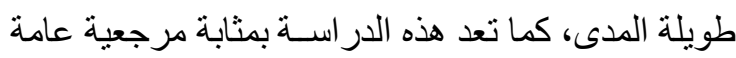
لمختلف الباحثين و الدارسين بالجامعات.

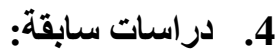

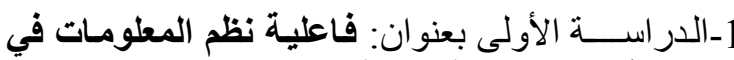

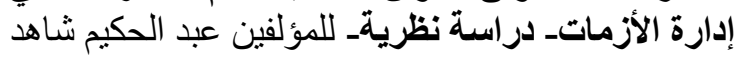

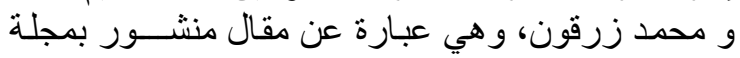
الدر اسـات الاقتصــادية و المالية بجامعة الو الو ادي الجز ائري

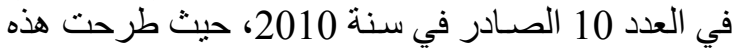

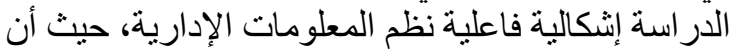

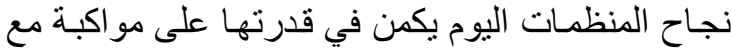

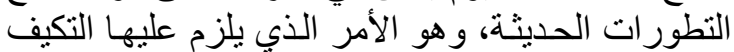

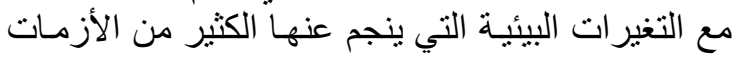




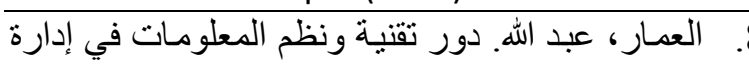
الأزمات و الكوارث، ماجســتير، العلوم الإدارية، كلية العادية الدراسـات العليا، أكاديمية نايف العربية للعلوم الأمنية، الأداتية .2012

9. شــــاهد، عبد الحكيم؛ زرقون، محمدــ فـاعليـة نظم

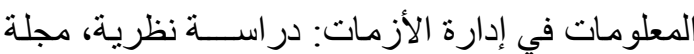

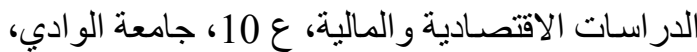
2017

10. شـريف، أنور ؛ عودة، بلال. دور تكنولوجيا المعلومات في الأداء الوظيفي، مجلة العلوم الاقتصادية و الإدارية،

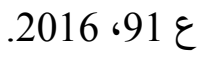
11. شـــرفا، محمد ســـلوى. إدارة المعلومات وتكنولوجيا

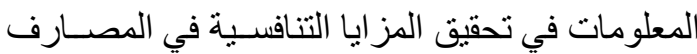

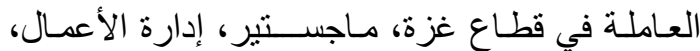
الجامعة الإسلامية، كلبة التجارة،

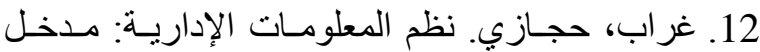
تحليلي، الرياض: مطابع جامعة الملك سعود، 1998.
1-تواجه الكلية عدة مخاطر تتظيمية، إدارية و علميـة في ظل جائحة كورونا

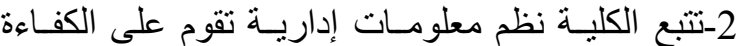

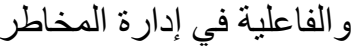
3-تختصـر نظم المعلومات الإدارية الوقت و الجهر وتكلفة إدارة المخاطر بالطرق التقليدية

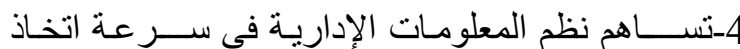
القرارات بثـــأن إدارة المخـاطر بأقل الإمكانيات و واقل الخسائر 5-تساهم نظم المعلومات الإدارية في إدارة جائحة كورونا

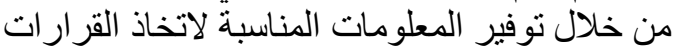

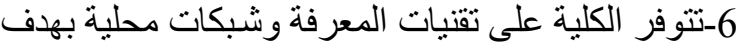

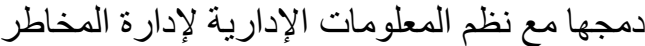

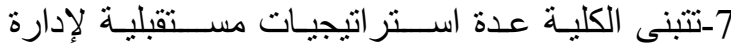

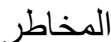

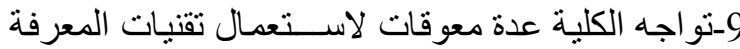
لإدارة المخاطر خلال الأزمات الرة الرات الهنة. 7. - ت توصيات:

1-ضـــرورة تطوير مهار ات اســتعمـال التقنيات الحديثة

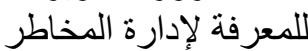
2-ضـرورة إحباء مختلف التظاهر ات العلمية والمعرفية في

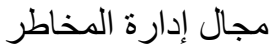

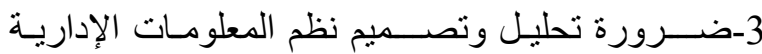
لاكتشاف مو اطن القوة و الضعف 4-حل مشــكلات نظم المعلومات الإدادارية و إدارة المخاطر على جميع الأصعدة.

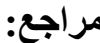

1. بغو، ألفة. دور إدارة المخاطر في تحقيق جودة خدمات

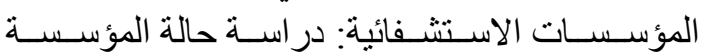

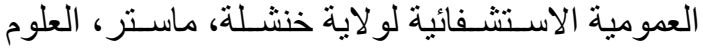

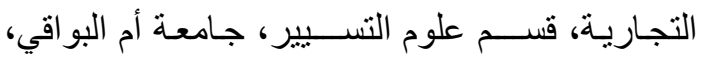
2013 2. الطائي، محمد. نظم المعلومات الإدارية، بغداد: جامعة

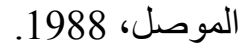

3. مهنـا، محمـــ. إدارة الأزمـات: قراءة في المنهج،

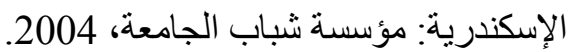

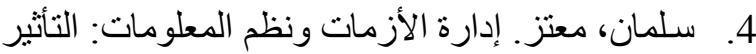

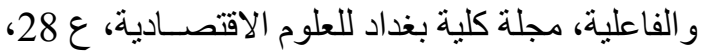

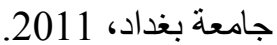
5. سـرور، علي. الذكاء الاصـطناعي، دليل النظم الذكية، الرياض: دار المريخ، 2005. 6. سلمان، معتز. إدارة الأزمات ونظم المعلومات: التأثير

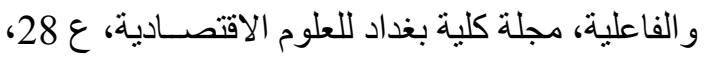
جامعة بغداد، 7. عبد المنعم، عاطف وآخرون. تقييم و إدارة المخاطر، جامعة القاهرة: كلية الهندسة، 2008. 